\title{
Sospecha clínica del trastorno de la deglución corroborado por fibroscopia endoscópica en pacientes críticos: análisis de utilidad y correlación de los factores de riesgo
}

Clinical suspicion of dysphagia confirmed by fiberoptic endoscopic evaluation in critical care patients: analysis of utility and correlation with risk factors Suspeita clínica de disfagia confirmada por fibroscopia endoscópica em pacientes em estado crítico: análise de utilidade e correlação com fatores de risco

Luis Ernesto Varela Sánchez, ${ }^{*}$ Luisa Irina Chávez Requena, ${ }^{*}$ Amparo Sumano Gotóo, ${ }^{*}$ Axel Pedraza Montenegro, ${ }^{*}$ Janet Silvia Sánchez Aguirre, ${ }^{*}$ Juvenal Franco Granillo*

\section{RESUMEN}

Introducción: Los trastornos de deglución son un factor importante de morbilidad y mortalidad; la valoración por fibroscopia endoscópica tiene ventajas tangibles contra otro tipo de estudios de la deglución. El paciente crítico con disfagia ha sido estudiado en relación con diferentes factores de riesgo como medicamentos, procedimientos invasivos y diferentes patologías, sin embargo, en pocos estudios se busca una relación directa con respecto al mecanismo causal del trastorno de deglución.

Objetivos: Establecer la relación entre diferentes factores de riesgo con los trastornos de la deglución en fase oral y faríngea, y con la presencia de penetración laríngea o aspiración traqueal en pacientes que han cursado con cuidados críticos; también valorar los factores de riesgo de acuerdo con el sustrato anatómico en el que se desarrolla.

Material y métodos: Realizamos un estudio observacional y retrolectivo de una cohorte; en este estudio se incluyeron exclusivamente pacientes internados en terapia intensiva e intermedia desde julio de 2016 hasta febrero de 2019 y mayores de 18 años, los cuales cursaron con valoración de la deglución por fibroscopia endoscópica.

Se realizó un análisis univariado de los factores seleccionados. El análisis estadístico se elaboró en SPSS v.21 y se analizaron las medidas de frecuencia; se realizó el análisis de los factores de riesgo con prueba de Fisher y $\chi^{2}$.

Resultados: Se realizó un total de 40 valoraciones por fibroscopia endoscópica; se confirmó algún trastorno de deglución en $82 \%$ de los pacientes, 18 del género masculino y 15 femenino; de éstos, 30\% tuvo penetración laríngea y $18 \%$ aspiración de contenido; en $60 \%$ hubo alteración de la fase oral y de la fase faríngea en $66 \%$ de los pacientes. Se asoció la penetración laríngea con el uso de sonda nasogástrica ( $\mathrm{p}$ 0.02), la aspiración traqueal con el uso de opioides ( $p 0.05$ ) y benzodiacepinas ( $p$ 0.05). En el caso de alteraciones de la fase oral se asoció el uso de opioides ( $p 0.03$ ), y en las alteraciones de la fase faríngea no se asoció con algún factor de riesgo específico.

Conclusión: El desarrollo de los trastornos de deglución en pacientes críticos no se relaciona con una sola causa, sino que se han asociado múltiples factores de riesgo en diferentes estudios, pero la sinergia no se ha asociado entre diferentes eventos y/o factores predisponentes. Debemos tomar en cuenta que diferentes factores de riesgo pueden tener un sustrato anatomofisiológico similar, lo que provoca que múltiples eventos resulten en un trastorno significativo de la deglución.

Palabras clave: Deglución, disfagia, fibroscopia.

\section{ABSTRACT}

Introduction: Swallowing disorders are an important factor of morbidity and mortality, endoscopic fibroscopic evaluation has tangible advantages agains other types of deglutition studies. The critically ill patient with dysphagia has been studied in relation to different risk factors such as medications, invasive procedures and different pathologies, however, in few studies a direct relationship is sought with respect to the causative mechanism of the swallowing disorder Objectives: To establish the relationship between different risk factors with dysphagia in the oral phase, the pharyngeal phase and with the presence of laryngeal penetration or tracheal aspiration in patients who have undergone

\footnotetext{
* Centro Médico ABC, Ciudad de México, México.
}

Recepción: 20/09/2019. Aceptación: 04/02/2020.

www.medigraphic.com/medicinacritica critical care and assess the risk factors according to the anatomical substrate in which it develops on.

Material and methods: We conducted a study of an observational, retrospective cohort, which exclusively included patients admitted to intensive and intermediate therapy from July 2016 to February 2019, of over 18 years of age, who were studied with endoscopic fibroscopy for dysphagia. A univariate analysis of selected factors was performed, the statistical analysis was prepared in SPSS v.21, frequency measurements were analyzed and the risk factor analysis was performed with Fisher test and $\chi^{2}$ test.

Results: A total of 40 evaluations were performed by endoscopic fibroscopy, a deglutition disorder was confirmed in $82 \%$ of the patients, 18 males and 15 females. 30\% had laryngeal penetration, 18\% had tracheal aspiration, $60 \%$ had alteration of the oral phase and $66 \%$ had alterations in the pharyngeal phase. Laryngeal penetration was associated with the use of nasogastric tubes ( $p$ $0.02)$, tracheal aspiration with the use of opioids ( $p 0.05$ ) and benzodiazepines (p 0.05). In the case of alterations of the oral phase, the use of opioids was associated ( $p$ 0.03) and in alterations of the pharyngeal phase there was no associated risk factor.

Conclusion: The development of deglutition disorders in critical patients is not related to a single cause, multiple risk factors have been associated in different studies but the synergy between different events and/or predisposing factors has not been associated. We must consider that different risk factors may have a similar anatomophysiological substrate, causing multiple events to result in a significant swallowing disorder.

Keywords: Deglutition, dysphagia, fibroscopy.

\section{RESUMO}

Introdução: Os distúrbios da deglutição são um importante fator de morbimortalidade; a avaliação por fibroscopia endoscópica apresenta vantagens tangíveis em relação a outros tipos de estudos da deglutição. $O$ paciente crítico com disfagia tem sido estudado em relação a diferentes fatores de risco, como medicamentos, procedimentos invasivos e diferentes patologias; no entanto, em alguns estudos busca-se uma relação direta com relação ao mecanismo causador do distúrbio da deglutição.

Objetivos: Estabelecer a relação entre diferentes fatores de risco com distúrbios da deglutição na fase oral, faríngea e com presença de penetração laríngea ou aspiração traqueal em pacientes submetidos a cuidados intensivos e avaliar os fatores de risco de acordo com o substrato anatômico em aquele que desenvolve.

Material e métodos: Realizamos um estudo de coorte, observacional, retroletivo, que incluiu exclusivamente pacientes admitidos na unidade de terapia intensiva e intermediária de julho de 2016 a fevereiro de 2019, com mais de 18 anos de idade que foram estudados com fibroscopia endoscópica da deglutição. Foi realizada uma análise univariada dos fatores selecionados; a análise estatística foi preparada no SPSS v.21, as medidas de frequência foram analisadas e a análise de fatores de risco foi realizada com o teste de Fisher $e \chi^{2}$.

Resultados: Foram realizadas 40 avaliações por fibroscopia endoscópica, sendo confirmados distúrbios da deglutição em $82 \%$ dos pacientes, 18 no masculino e 15 no feminino. 30\% apresentaram penetração laríngea, 18\% aspiração de conteúdo, alteração da fase oral em $60 \%$ e fase faríngea em $66 \%$ dos pacientes. A penetração laríngea foi associada ao uso de sonda nasogástrica ( $p$ 0.02), aspiração traqueal ao uso de opioides ( $p 0.05)$ e benzodiazepínicos ( $p$ 0.05). No caso de alterações da fase oral, associouse o uso de opioides ( $p$ 0.03) e nas alterações da fase faríngea, não esteve associado a um fator de risco específico. 
Conclusão: O desenvolvimento dos distúrbios da deglutição em pacientes críticos não está relacionado a uma única causa, múltiplos fatores de risco foram associados em diferentes estudos, mas a sinergia entre diferentes eventos e/ou fatores predisponentes não foi associada. Devemos considerar que diferentes fatores de risco podem ter um substrato anatomofisiológico semelhante, causando vários eventos que resultam em um distúrbio significativo da deglutição. Palavras-chave: Deglutir, disfagia, fibroscopia.

\section{INTRODUCCIÓN}

Los trastornos de la deglución son un factor importante de morbilidad y mortalidad en pacientes críticos; consecuentemente, éstos son una causa de mayor estancia en terapia intensiva y, de esta manera, conllevan un mayor costo hospitalario. ${ }^{1,2}$ La prevalencia de disfagia puede oscilar de 30 a $60 \%$, por lo que su diagnóstico temprano puede ayudar a evitar complicaciones y potencialmente mejorar el desenlace en el ámbito nutricional y respiratorio. ${ }^{3}$

La valoración de la deglución implica determinar si hay alteración de ésta y, de ser así, comprobar los grados en los que se encuentran las deficiencias funcionales y la capacidad del paciente para compensarlos. ${ }^{4} \mathrm{La}$ indicación del tipo de valoración de la deglución a realizar normalmente recae en el clínico y depende de los antecedentes del paciente, de los elementos diagnósticos con los que se cuente y del protocolo hospitalario.

Dentro de la evaluación de los trastornos de la deglución en el paciente crítico, se han descrito varias opciones para determinar la seguridad de inicio de la vía oral después de un proceso de enfermedad aguda. Dentro de estas opciones, comúnmente están descritas la valoración clínica a la cabecera del paciente, la videofluoroscopia de la deglución y la valoración por fibroscopia endoscópica.

La valoración clínica de la deglución a la cabecera del paciente es el recurso más usado; esta prueba consiste en la administración de $10 \mathrm{~mL}$ de líquido y, posteriormente, se observan la presencia de secreciones orales, el intento por deglutir, la presencia de tos y la dificultad para el habla. Esta prueba conlleva riesgos y desventajas importantes, además, no determina de manera objetiva la respuesta a diferentes consistencias alimenticias, depende de la presencia de un reflejo tusígeno intacto para que su interpretación sea concordante con la presencia o no de broncoaspiración, no detecta la cantidad de residuo en estructuras orofaríngeas, no puede valorar la respuesta a diferentes maniobras compensatorias y tiene el riesgo de que el paciente broncoaspire durante la valoración, con las diferentes complicaciones que esto representa.

La videofluoroscopia se ha usado como la prueba de referencia para determinar la presencia de aspiración por medio de la visualización del paso del contenido alimenticio en tiempo real y tiene ventajas tangibles con respecto al estudio clínico que está a la cabecera del paciente. La endoscopia o fibroscopia nasal tiene múlti- ples puntos a su favor; por ejemplo, tiene la capacidad de distinguir la presencia de trastornos de deglución, aspiración, penetración laríngea, residuo excesivo y visualizar las alteraciones a nivel de cuerdas vocales; 5 además, el médico que realiza el estudio tiene la capacidad de controlar la cantidad de material alimenticio que se usa en la prueba y evitar eventos significativos de aspiración. Este estudio, que se desarrolló desde 1988, consiste en la introducción de un fibroscopio vía nasal para la visualización de las estructuras orofaríngeas y laríngeas; así se valora la respuesta deglutoria a diferentes consistencias de alimento y a diferentes respuestas y maniobras de compensación. Hasta hace unos años, varías guías de práctica clínica no tomaban en cuenta a la fibroscopia endoscópica para la valoración de la deglución, sin embargo, se ha comparado su efectividad con respecto a la videofluoroscopia y cuenta con una adecuada concordancia interobservador. ${ }^{3}$

\section{Anatomía y fisiología de la deglución}

La deglución es un acto neuromuscular complejo que implica una interacción coordinada de diversas estructuras de la vía aerodigestiva, que están localizadas en la cabeza, cuello y tórax. Para entender los trastornos de la deglución se deben cubrir las bases anatomofisiológicas de las estructuras que asisten en ella.

La laringe se divide en supraglotis, glotis y subglotis; dentro de sus funciones más importantes, se encuentran la protección de la vía aérea durante la deglución y realizar la fonación. Conforme aumenta la edad, ocurre un cambio progresivo en ciertas estructuras de la laringe que pueden afectar la cinemática de la deglución. ${ }^{6}$ La glotis corresponde al plano formado entre las cuerdas vocales, aritenoides y comisura anterior y posterior. La musculatura laríngea se puede dividir en extrínseca e intrínseca, dependiendo de si una o ambas inserciones se localizan en la laringe; los músculos intrínsecos se encuentran a cargo de la aducción, abducción y la tensión de las cuerdas vocales, mientras que los músculos extrínsecos levantan la laringe desplazándola para poder bloquear.

Desde un punto de vista motor, todos los músculos intrínsecos son inervados por el nervio laríngeo recurrente, a excepción del músculo cricotiroideo que es inervado por el nervio laríngeo superior. La inervación sensitiva de la supraglotis y glotis se lleva por los nervios laríngeo superior y laríngeo recurrente. ${ }^{7}$ Durante la respiración las cuerdas vocales se abren; ésta es su posición en reposo. Durante la deglución la laringe se eleva y las cuerdas vocales se cierran momentáneamente, bloqueando así el flujo hacia el plano glótico..$^{3,8}$

La deglución se puede dividir en fase preparatoria, oral, faríngea y esofágica; cada una de estas fases puede afectarse de forma distinta, lo que resulta en disfagia, aspiración y regurgitación. La presencia 
de penetración laríngea supone la entrada de alimento al vestíbulo laríngeo y la aspiración supone la entrada de alimento en la laringe. La fase preparatoria y oral implican la masticación y transferencia del alimento y la fase faríngea requiere una función adecuada a nivel velofaríngeo, lingual, hipofaríngeo y laríngeo; las bandas ventriculares y las cuerdas vocales se cierran, la epiglotis se desplaza de manera posterior, la laringe se eleva y se contrae la faringe. Durante la fase esofágica el esfínter esofágico inferior se relaja y permite el paso del bolo a la cámara gástrica. ${ }^{9}$

Para el control central de la deglución se deben coordinar acciones reflejas para proteger la vía aérea, pero también se deben llevar a cabo acciones motoras voluntarias, además de la coordinación adecuada de las vías motoras y sensitivas; a nivel cortical y subcortical, éstas son indispensables para mantener una deglución adecuada. ${ }^{8}$

\section{Trastornos de la deglución en pacientes críticos}

Existen factores que se han asociado con alteraciones de la deglución en pacientes críticos; entre éstos se encuentran la intubación orotraqueal, el uso de bloqueadores neuromusculares y/o sedantes, la edad, la alteración del estado de alerta, delirio, debilidad adquirida, polineuropatía del enfermo en estado crítico, trastornos neurológico previos o adquiridos, la presencia de traqueostomía, globo de traqueostomía demasiado inflado y la presencia de sonda nasogástrica, entre otros. ${ }^{10,11}$

No hay estudios enfocados a pacientes críticos que determinen la frecuencia de broncoaspiración en relación con diferentes factores de riesgo y tampoco se ha establecido el estudio ideal para valorar a esta población, sin embargo, en un estudio realizado por Ponfick M y colaboradores, en el 2015, se valoró la deglución mediante endoscopia en una cohorte de 22 pacientes con polineuropatía del paciente crítico a los 3, 14 y 28 días después de la admisión a terapia intensiva; en este estudio se encontró una tasa de aspiración de $78 \%$ con líquidos y saliva; por su parte, en alimentos con consistencia de puré, se observó seguridad en la deglución en $82 \%$ de los casos. ${ }^{12}$ Dichos hallazgos tienen una alta relevancia clínica, ya que el inicio temprano de la dieta enteral se puede dar de manera segura sin necesidad de recurrir a procedimientos como la colocación de sondas nasogástricas o gastrostomías; así, se da tiempo para continuar con terapia de deglución, buscando corregir las alteraciones que inicialmente llevaron al trastorno de la deglución.

Se han enfocado los estudios sobre trastornos de la deglución en subgrupos de pacientes críticos como es el caso de pacientes con sepsis, en los cuales se encontró que, de acuerdo con la clasificación previa de sepsis, los pacientes con diagnóstico de sepsis severa tuvieron una mayor frecuencia de disfagia después de 14 días de estancia en la Unidad de Cuidados Críticos y cuatro meses después de la instauración de la enfermedad crítica, a diferencia de pacientes sin sepsis severa. ${ }^{1}$

No hay estudios que valoren la incidencia de trastornos de la deglución en presencia de diferentes factores de riesgo; la mayoría de los estudios se centran en factores específicos sin hacer una comparación de la frecuencia de éstos, pero tampoco se han analizado diferentes factores en subpoblaciones de pacientes críticos.

El objetivo de este estudio es establecer la relación entre diferentes factores de riesgo con los trastornos de la deglución en fase oral, faríngea y con la presencia de penetración laríngea o aspiración traqueal en pacientes que han cursado con cuidados críticos, así como valorar los factores de riesgo de acuerdo con el sustrato anatómico en el que se desarrolla.

\section{MATERIAL Y MÉTODOS}

Realizamos un estudio de una cohorte, observacional y retrolectivo, en el que se incluyeron exclusivamente pacientes internados en terapia intensiva e intermedia, que fueran mayores de 18 años y que cursaran con

Tabla 1: Características demográficas basales de población con trastorno de la deglución confirmado.

\begin{tabular}{|c|c|c|c|c|}
\hline Variable & $\begin{array}{l}\text { Pacientes con sospecha clínica } \\
\text { de trastorno de deglución }\end{array}$ & $\begin{array}{l}\text { Trastorno de la deglución } \\
\text { por endoscopia negativo }\end{array}$ & $\begin{array}{c}\text { Trastorno de la deglución } \\
\text { confirmado }\end{array}$ & $\mathrm{p}$ \\
\hline \multicolumn{5}{|l|}{ Género (\%) } \\
\hline Masculino & $17(42.5)$ & $2(5.0)$ & $18(45.0)$ & 0.41 \\
\hline Edad media & 65 & 64.5 & 65.5 & 0.76 \\
\hline \multicolumn{5}{|l|}{ Motivo principal de ingreso (\%) } \\
\hline Vigilancia neurológica & $26(65.0)$ & $5(12.5)$ & $21(52.5)$ & 0.34 \\
\hline \multicolumn{5}{|l|}{ Etiología patológica principal (\%) } \\
\hline Sepsis & $9(22.5)$ & $1(2.5)$ & $8(20.0)$ & 0.24 \\
\hline Trastorno neurológico no asociado a trauma & $23(57.5)$ & $3(7.5)$ & $20(50.0)$ & \\
\hline Trauma & $8(20.0)$ & $3(7.5)$ & $5(12.5)$ & \\
\hline Ventilación mecánica (\%) & 26 & $6(16.0)$ & $20(50.0)$ & 0.2 \\
\hline
\end{tabular}


Tabla 2: Trastornos de la deglución a razón de factores de riesgo.

\begin{tabular}{lcc}
\hline & $\begin{array}{c}\text { Trastorno de } \\
\text { la deglución } \\
\text { confirmado, } \mathrm{n}(\%)\end{array}$ & $\mathrm{p}$ \\
\hline Reintubación & $2(5.0)$ & 0.5 \\
Lesión laríngea & $2(5.0)$ & 0.5 \\
Traqueostomía & $4(10.0)$ & 0.27 \\
Extubación no planeada & $2(5.0)$ & 0.5 \\
Días en ventilación invasiva (media) & 5.2 días & 0.37 \\
Trastorno neurológico previo & $29(72.5)$ & 0.05 \\
Sonda nasogástrica & $20(50.0)$ & 0.86 \\
Sonda orogástrica & $8(20.0)$ & 0.08 \\
Delirio & $13(32.5)$ & 0.86 \\
Uso de propofol > 0.4 mg/kg/día & $2(5.0)$ & 0.03 \\
Uso de opioides & $15(37.5)$ & 0.21 \\
Uso de benzodiacepinas & $15(37.5)$ & 0.09 \\
Bloqueador neuromuscular & $4(10.0)$ & 0.44 \\
\hline
\end{tabular}

valoración de la deglución por fibroscopia endoscópica desde julio de 2016 a febrero de 2019.

Se realizó un análisis univariado de los factores seleccionados y se relacionó la presencia de trastornos de deglución, de acuerdo con las alteraciones en la fase oral o faríngea, y en la presencia de aspiración o penetración laríngea. El análisis estadístico se elaboró en SPSS v.21; se analizaron las medidas de frecuencia y se realizó el análisis de los factores de riesgo con prueba de Fisher y $\chi^{2}$.

\section{RESULTADOS}

Se valoró por fibroscopia endoscópica a un total de 41 pacientes con sospecha de trastorno de deglución y se excluyó un paciente al no poder realizarse el estudio de manera adecuada. Dentro de las 40 valoraciones por fibroscopia endoscópica se confirmó algún trastorno de deglución en $82 \%$ de los pacientes, 18 del género masculino y 15 femenino.

De los pacientes valorados por endoscopia que tuvieron algún grado de trastorno de la deglución, 30\% tuvieron penetración laríngea y $18 \%$ aspiración de contenido. Se encontró además, alteración de la fase oral en $60 \%$ de los pacientes y de la fase faríngea en $66 \%$ de los pacientes.

El motivo principal de ingreso a terapia intensiva fue el mantener vigilancia neurológica en $52.5 \%$ de los pacientes, continuar con el apoyo ventilatorio en $17.5 \%$ y monitoreo hemodinámico en $12.5 \%$. Dentro de la etiología patológica principal de los pacientes se encontró que $57.5 \%$ ingresaron por trastornos neurológicos no asociados a trauma, $22.5 \%$ por sepsis y $20 \%$ por causas asociadas a trauma. De los 33 pacientes con trastorno de deglución se encontró que 20 cursaron con ventilación mecánica invasiva (p 0.2) (Tabla 1).

Dentro de los factores de riesgo valorados, se tomaron en cuenta la presencia de sepsis o choque séptico, el uso de sonda orogástrica o nasogástrica, la presencia de traqueostomía, el uso previo a la prueba de propofol con una dosis mayor a $4 \mathrm{mg} / \mathrm{kg} / \mathrm{hora}$, el uso de benzodiacepinas y opioides previo o durante la prueba, el uso previo a la prueba de bloqueo neuromuscular en infusión y antecedente de trastorno neurológico estructural o funcional, reintubación, lesión laríngea documentada al momento de la intubación, extubación no planeada, los días totales en ventilación mecánica invasiva y la presencia de delirio.

Dentro de los factores de riesgo analizados se encontró que los pacientes con trastorno neurológico previo ( $p$ 0.05) tuvieron mayor frecuencia de trastornos de la deglución en general, al igual que los pacientes que cursaron con uso de propofol a dosis $>4 \mathrm{mg} / \mathrm{kg} / \mathrm{hora}$ ( $\mathrm{p}$ 0.03 ) independiente del tiempo de infusión (Tabla 2).

Tabla 3: Trastornos de la deglución ajustada a estado.

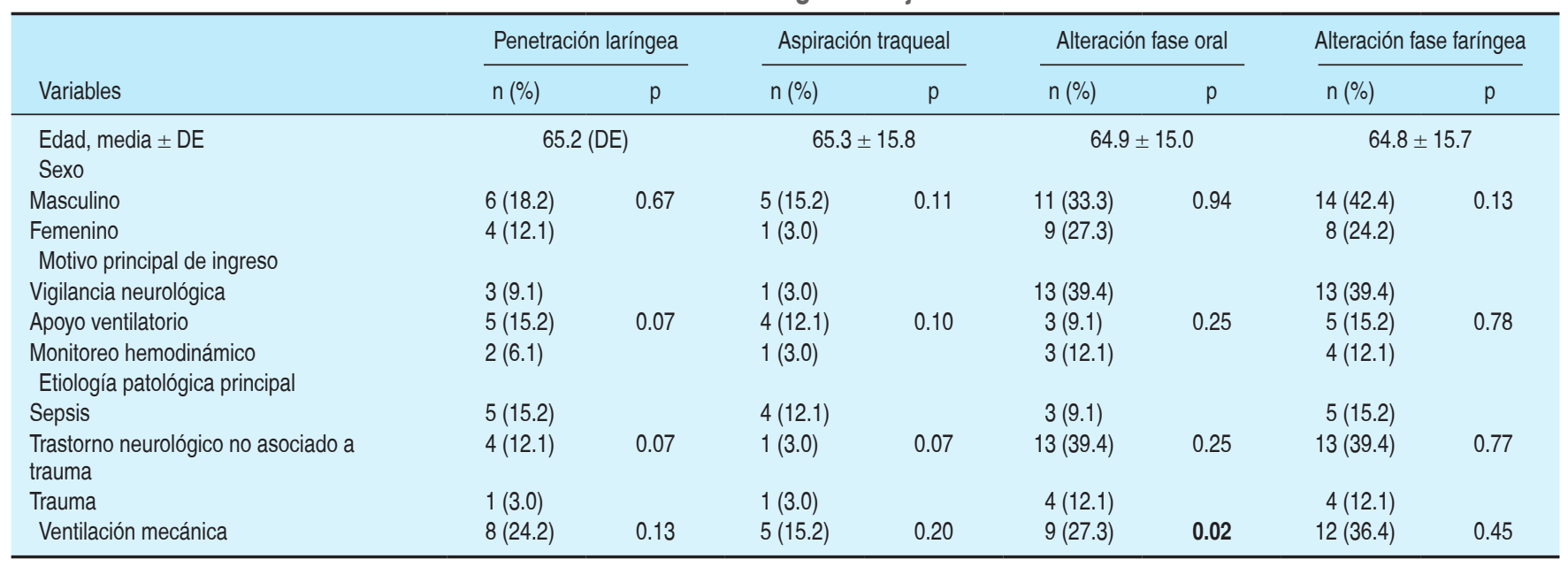

$\mathrm{DE}=$ Desviación estándar. 
Tabla 4: Trastorno de la deglución y factores de riesgo.

\begin{tabular}{|c|c|c|c|c|c|c|c|c|}
\hline \multirow[b]{2}{*}{ Variables } & \multicolumn{2}{|c|}{ Penetración laríngea } & \multicolumn{2}{|c|}{ Aspiración traqueal } & \multicolumn{2}{|c|}{ Alteración fase oral } & \multicolumn{2}{|c|}{ Alteración fase faríngea } \\
\hline & $\mathrm{n}(\%)$ & $\mathrm{p}$ & $\mathrm{n}(\%)$ & $p$ & $\mathrm{n}(\%)$ & $p$ & $n(\%)$ & $p$ \\
\hline Reintubación & $3(9.1)$ & 0.07 & $1(3.0)$ & 0.70 & $1(3.0)$ & 0.12 & $3(9.1)$ & 0.70 \\
\hline Lesión laríngea & $1(3.0)$ & 0.52 & 0 & 0.66 & $1(3.0)$ & 0.64 & $1(3.0)$ & 0.56 \\
\hline Traqueostomía & $2(6.1)$ & 0.35 & $1(3.0)$ & 0.57 & $2(6.1)$ & 0.51 & $4(12.1)$ & 0.17 \\
\hline Extubación no planeada & $2(6.1)$ & 0.08 & $1(3.0)$ & 0.33 & $1(3.0)$ & 0.64 & $2(6.1)$ & 0.54 \\
\hline Trastorno neurológico previo & $3(9.1)$ & 0.14 & $2(6.1)$ & 0.21 & $5(15.2)$ & 0.06 & $5(15.2)$ & 0.11 \\
\hline Sonda nasogástrica & $9(27.3)$ & 0.02 & $5(15.2)$ & 0.36 & $12(36.4)$ & 0.61 & $15(45.5)$ & 0.18 \\
\hline Sonda orogástrica & $2(6.1)$ & 0.53 & 0 & 0.16 & $4(12.1)$ & 0.38 & 5 (15.2) & 0.54 \\
\hline Delirio & $6(18.2)$ & 0.11 & $6(18.2)$ & 0.14 & $6(18.2)$ & 0.15 & $7(21.2)$ & 0.18 \\
\hline Uso de propofol & $1(3.0)$ & 0.52 & $1(3.0)$ & 0.33 & 0 & 0.14 & $1(3.0)$ & 0.56 \\
\hline Uso de opioides & $6(18.2)$ & 0.06 & $5(15.2)$ & 0.05 & $6(18.2)$ & 0.03 & $8(24.2)$ & 0.13 \\
\hline Uso de benzodiacepinas & $2(6.1)$ & 0.60 & $3(9.1)$ & 0.05 & $1(3.0)$ & 0.06 & $3(9.1)$ & 0.30 \\
\hline Bloqueador neuromuscular & $3(9.1)$ & 0.07 & $2(6.1)$ & 0.14 & $1(3.0)$ & 0.15 & $4(12.1)$ & 0.17 \\
\hline
\end{tabular}

Con respecto a las alteraciones específicas de la deglución, no se relacionaron la penetración laríngea ni la aspiración traqueal, ni las alteraciones en fase faríngea con alguna variable relacionada al estado basal. Sin embargo, en el caso de alteraciones en fase oral se encontró que los pacientes que cursaron con ventilación mecánica presentaron una mayor frecuencia de alteraciones en esta fase (Tabla 3).

Dentro de los factores de riesgo específicos, se asoció la penetración laríngea con el uso de sonda nasogástrica ( $p 0.02$ ); la aspiración traqueal se relacionó con el uso de opioides ( $p 0.05$ ) y benzodiacepinas ( $p 0.05$ ). El opioide más usado en nuestros pacientes fue fentanil y las benzodiacepinas más usadas incluyeron midazolam y lorazepam. En el caso de alteraciones de la fase oral se asoció nuevamente el uso de opioides ( $p 0.03$ ) y en el caso de las alteraciones de la fase faríngea no se asoció ningún factor de riesgo específico (Tabla 4).

\section{DISCUSIÓN}

Los resultados de nuestro estudio no confirman la correlación de diferentes factores de riesgo descritos en la literatura, sólo se relaciona el uso de sedantes, opiáceos, benzodiacepinas y el uso de sonda orogástrica con alteraciones en diferentes fases de la deglución y en la penetración laríngea y aspiración. A pesar de que en nuestra valoración inicial se detectó que los pacientes con trastorno neurológico previo y con el uso de propofol eran los que tenían una frecuencia significativa de trastornos de deglución, ninguno de estos factores reflejó una alteración significativa en la presencia de aspiración traqueal, penetración laríngea o alteraciones en las fases oral y faríngea.

En el caso de factores de riesgo como la reintubación, lesión laríngea, uso de bloqueador neuromuscular y traqueostomía, se observa que nuestra población es pequeña, por lo que nuestro análisis estadístico tiene limitada capacidad para discernir adecuadamente el efecto potencial que estos factores pudieran tener en la deglución.

A pesar de que en nuestro estudio se relacionaron algunos factores de riesgo con ciertas alteraciones de la deglución, no se tuvo una correlación importante en la mayoría de las fases. Debemos pensar entonces que los trastornos neuromusculares que provocan estas alteraciones son el resultado de eventos traumáticos iatrogénicos y no iatrogénicos como pueden ser la intubación, la colocación de sondas, el efecto deletéreo que tienen los procesos sépticos o la neuropatía del enfermo en estado crítico, el uso de sedantes, analgésicos y bloqueadores neuromusculares.

Se deben realizar estudios con mayor cantidad de pacientes y relacionar diferentes factores de riesgo para tener evidencia más contundente sobre el origen anatomofisiológico de los trastornos de deglución que se encuentran en los estudios de fibroscopia endoscópica, sin embargo, no podemos descartar la importancia de esta prueba, ya que a diferencia de la valoración de la deglución a la cabecera del paciente y de la mecánica de deglución por videofluoroscopia, los datos que nos aporta este estudio son imprescindibles para empezar a comprender las razones del desarrollo de disfagia en nuestros pacientes críticos y para dar un adecuado manejo nutricional de manera temprana, buscando limitar el uso de sondas y otros procedimientos que son potencialmente innecesarios en el contexto de un estudio de la deglución por fibroscopia endoscópica.

\section{CONCLUSIONES}

El desarrollo de los trastornos de deglución en pacientes críticos no se relaciona con una sola causa; se han asociado múltiples factores de riesgo en diferentes estudios, pero no se ha asociado la sinergia entre diferentes eventos y/o factores predisponentes. Debemos tomar en cuenta que diferentes factores de riesgo pueden tener un sustrato anatomofisiológico similar, provo- 
cando que múltiples eventos resulten en un trastorno significativo de la deglución.

Se deben realizar estudios subsecuentes para determinar la potencial interacción de múltiples factores de riesgo en los trastornos de la deglución y determinar qué peso tienen en relación con el inicio de nutrición óptima y de potenciales complicaciones respiratorias.

Es importante contar con un médico foniatra capaz de realizar valoraciones por fibroscopia endoscópica en pacientes que han cursado con cuidados críticos y que se consideran en riesgo de desarrollo de trastornos de la deglución; su implementación en las Unidades de Cuidados Intensivos ha sido lenta, pero el beneficio potencial, que tiene este tipo de pruebas, puede cambiar el desenlace de algunos de nuestros pacientes.

\section{BIBLIOGRAFÍA}

1. Zielske J, Bohne S, Brunkhorst FM, Axer H, Guntinas-Lichius O. Acute and long-term dysphagia in critically ill patients with severe sepsis: results of a prospective controlled observational study. Eur Arch Otorhinolaryngol. 2014;271(11):3085-3093.

2. Padovani AR, Moraes DP, Sassi FC, Andrade CR. Avaliação clínica da deglutição em unidade de terapia intensiva. CoDAS. 2013;25(1):1-7.

3. Clavé P, Arreola A, Velasco M, Puiggros C. Abordaje clínico de la disfagia orofaríngea: diagnóstico y tratamiento. Nutr Cl. 2007;1(3):174-182.

4. Carnaby-Mann G, Lenius K. The bedside examination in dysphagia. Phys Med Rehabil Clin N Am. 2008;19(4):747-768, viii.

5. Langmore SE, Schatz K, Olson N. Endoscopic and videofluoroscopic evaluations of swallowing and aspiration. Ann Otol Rhinol Laryngol. 1991;100(8):678-681.
6. Inamoto Y, Saitoh E, Okada S, Kagaya H, Shibata S, Baba M, et al. Anatomy of the larynx and pharynx: effects of age, gender and height revealed by multidetector computed tomography. $J$ Oral Rehabil. 2015;42(9):670-677.

7. García-Araque HF, Gutiérrez-Vidal SE. Aspectos básicos del manejo de la vía aérea: anatomía y fisiología. Rev Mex Anest. 2015;38(2):98-107.

8. Sasegbon A, Hamdy S. The anatomy and physiology of normal and abnormal swallowing in oropharyngeal dysphagia. Neurogastroenterol Motil. 2017;29(11).

9. Alvo A, Olavarría C. Decannulation and assessment of deglutition in the tracheostomized patient in non-neurocritical intensive care. Acta Otorrinolaringol Esp. 2014;65(2):114-119.

10. Huggins PS, Tuomi SK, Young C. Effects of nasogastric tubes on the young, normal swallowing mechanism. Dysphagia. 1999;14(3):157-161.

11. Amathieu R, Sauvat S, Reynaud P, Slavov V, Luis D, Dinca A et al. Influence of the cuff pressure on the swallowing reflex in tracheostomized intensive care unit patients. $\mathrm{Br} J$ Anaesth. 2012;109(4):578-583.

12. Ponfick M, Linden R, Nowak DA. Dysphagia--a common, transient symptom in critical illness polyneuropathy: a fiberoptic endoscopic evaluation of swallowing study*. Crit Care Med. 2015;43(2):365-372.

Patrocinios y relación de conflicto de intereses: No se cuenta con patrocinio público o privado para la elaboración de este estudio.

\author{
Correspondencia: \\ Dr. Luis Ernesto Varela Sánchez \\ Sur 136 Núm. 116, \\ Col. Las Américas, 01120, \\ Álvaro Obregón, Ciudad de México. \\ Teléfono: 5320-800, ext. 2052 \\ E-mail: luisvarela86@gmail.com
}

